\title{
Recurrent Candida esophagitis in an Immunocompetent Patient
}

\author{
Mona Al Fares MD¹, Mohammed Barawi MD², Majeed Paduvana MD $^{3}$, Leonard B. Johnson MD FACP \\ Department of Internal Medicine, St John Hospital and Medical Center, Detroit, Michigan \\ Department of Internal Medicine, King Abdulaziz University Hospital, Jeddah, Saudi Arabia
}

Keywords: Candida, fungal, infection, esophagitis, biopsy

\section{Introduction}

Candida esophagitis (CE) is commonly reported in patients with impaired cell-mediated immunodeficiency, such as patients with AIDS and transplants, but rarely reported in immunocompetent adults. We report a case of recurrent Candida albicans esophagitis in a patient without traditional risk factors.

\section{Case}

A 72-year-old female with past medical history of insulinrequiring diabetes mellitus and mild asthma presented in September 2008 with progressive odynophagia and retrosternal chest discomfort for several months. The patient had lost five pounds but otherwise denied fevers, chills or other complaints. She had not taken systemic or inhaled corticosteroids or antibiotics in several years. Upon examination, she was well appearing, her mucous membranes were clear, there was no lymphadenopathy and her abdominal examination was within normal limits. Her CBC revealed a normal leukocyte count, hemoglobin 10.2 $\mathrm{g} / \mathrm{dL}$, albumin $3.1 \mathrm{~g} / \mathrm{dL}$ and HgbAlc 7.7\%. HIV antibody An esophagoduodenoscopy (EGD) was performed and revealed white exudate covering most of the esophagus. Esophageal manometry also revealed esophageal motility disorder. The patient was treated with a 10-day course of oral fluconazole with clinical resolution. Her symptoms recurred in May 2010 and repeat EGD again was consistent with CE both on appearance and in the biopsy. An HIV antibody was negative and quantitative serum immunoglobulins were within normal limits. The patient was treated again successfully with two weeks of oral fluconazole but she relapsed two weeks after discontinuing therapy. Over the last year, the patient has required suppressive fluconazole $100 \mathrm{mg}$ daily to prevent further relapses. In April 2011, she presented again with recurrent esophageal symptoms and repeat EGD again demonstrated findings consistent with CE. An esophageal biopsy was sent for fungal culture that grew $C$. albicans that demonstrated a fluconazole minimum inhibitory concentration of $16 \mu \mathrm{g} / \mathrm{mL}$ (susceptible $\leq 8$ ). The patient is now being treated with a fluconazole dose of $200 \mathrm{mg}$ daily pending further evaluation.

\section{Discussion}

This case illustrates that idiopathic CE can present in patients without traditional risk factors. Risk factors that have been identified among non-HIV and non-transplant patients include recent antibiotics, systemic or inhaled corticosteroids, diabetes mellitus, acid suppressive therapy and esophageal motility disorders. Our patient is unique with respect to the multiple recurrences and development of increasing azole resistance that are typically seen in patients with AIDS-related CE.

\section{References}

[1] Baehr PH, McDonald GB. Esophageal infections: risk factors, presentation, diagnosis, and treatment. Gastroenterology 1994; 106:509-32.

[2] Simon MR, Houser WL, Smith KA, Long PM. Esophageal candi- diasis as a complication of inhaled corticosteroids. Ann Allergy Asthma Immunol 1997;79:333-8.

[3] Phaosawasdi K, Rice P, Lee B. Primary and secondary Candida esophagitis. IMJ Ill Med J 1986;169:361-5.

[4] Mathieson R, Dutta SK. Candida esophagitis. Dig Dis Sci 1983;28:365-70.

[5] Yonsei Med J. 2013 Jan 1; 54(1): 160165. PMCID: PMC3521253

[6] M Baig, J Rasheed, D Subkowitz, J Vieira, S Gerges. Severe Esophageal Candidiasis In An Immunocompetent Patient. The Internet Journal of Infectious Diseases. 2005 Volume 5 Number 1. 